\title{
Cholesterol Defect Is Marked across Multiple Rodent Models of Huntington's Disease and Is Manifest in Astrocytes
}

\author{
Marta Valenza, ${ }^{1,2}$ Valerio Leoni, ${ }^{3 \star}$ Joanna M. Karasinska, ${ }^{4 \star}$ Lara Petricca, ${ }^{1,2 \star}$ Jianjia Fan, ${ }^{5}$ Jeffrey Carroll, ${ }^{4}$ \\ Mahmoud A. Pouladi, ${ }^{4}$ Elisa Fossale, ${ }^{6}$ Huu Phuc Nguyen, ${ }^{7}$ Olaf Riess, ${ }^{7}$ Marcy MacDonald, ${ }^{6}$ Cheryl Wellington, ${ }^{5}$ \\ Stefano DiDonato, ${ }^{3}$ Michael Hayden, ${ }^{4}$ and Elena Cattaneo ${ }^{1,2}$ \\ ${ }^{1}$ Department of Pharmacological Sciences and ${ }^{2}$ Centre for Stem Cell Research, Università degli Studi di Milano, 20133 Milan, Italy, ${ }^{3}$ Unit of Genetics of \\ Neurodegenerative and Metabolic Diseases, Carlo Besta Neurological Institute, 20133 Milan, Italy, ${ }^{4}$ Centre for Molecular Medicine and Therapeutics, \\ University of British Columbia, Vancouver, British Columbia, Canada V5Z 4H4, ${ }^{5}$ Department of Pathology and Laboratory Medicine, University of British \\ Columbia, Vancouver, British Columbia, Canada V6T 2B5, ${ }^{6}$ Centre for Human Genetic Research, Massachusetts General Hospital, Boston, Massachusetts \\ 02114, and 7Department of Medical Genetics, University of Tübingen, 72016 Tübingen, Germany
}

Brain cholesterol, which is synthesized locally, is a major component of myelin and cell membranes and participates in neuronal functions, such as membrane trafficking, signal transduction, neurotransmitter release, and synaptogenesis. Here we show that brain cholesterol biosynthesis is reduced in multiple transgenic and knock-in Huntington's disease (HD) rodent models, arguably dependent on deficits in mutant astrocytes. Mice carrying a progressively increased number of CAG repeats show a more evident reduction in cholesterol biosynthesis. In postnatal life, the cholesterol-dependent activities of neurons mainly rely on the transport of cholesterol from astrocytes on ApoE-containing particles. Our data show that mRNA levels of cholesterol biosynthesis and efflux genes are severely reduced in primary HD astrocytes, along with impaired cellular production and secretion of ApoE. Consistently, in CSF of HD mice, ApoE is mostly associated with smaller lipoproteins, indicating reduced cholesterol transport on ApoE-containing lipoproteins circulating in the HD brain. These findings indicate that cholesterol defect is robustly marked in HD animals, implying that strategies aimed at selectively modulating brain cholesterol metabolism might be of therapeutic significance.

\section{Introduction}

Huntington's disease (HD) is a progressive, neurodegenerative disorder caused by a CAG repeat expansion within the coding region of the HD gene (The Huntington's Disease Collaborative Research Group, 1993), resulting in an expanded glutamine tract in huntingtin, an ubiquitously expressed protein with beneficial activities for brain neurons (Zuccato et al., 2010). Patients with HD show predominant brain manifestations, with the striatal medium size spiny neurons and cortical neurons being particularly vulnerable (Vonsattel, 2008). The lack of appropriate disease models capable of faithfully recapitulating the entire life-long disease stages generates difficulties in the interpretation of under-

\footnotetext{
Received Feb. 19, 2010; revised June 12, 2010; accepted June 17, 2010.

This work was supported by CHDI Foundation (United States), STEM-HD (LHSB-CT-2006-037349, Sixth Framework Program, European Union), and Telethon (\#GGP02215) to E.C.; Huntington's Disease Society of America Coalition for the Cure (United States) to E.C., M.M., and M.H.; a National Institutes of Health grant (NS32765) to M.M.; and Fondazione Cariplo (Italy) to M.V. and M.H. E.C., M.M., and M.H. are members of the HDSA Coalition for the Cure "Huntingtin Function" Team. We thank Veronica Hirsch-Reinshagen for insightful comments and suggestions related to ApoE secretion in astrocytes and CHDI members for their continuous encouragement to investigate cholesterol dysfunction in HD. We are also very grateful to N. Bissada, L. Liu, Y. Xie (Robert), and P. Ruddle for their technica support during the time during which M.V. visited M.H.'s laboratory. Finally, we also thank the families of the patients with Huntington's disease for their continuous support.

*V.L., J.M.K., and L.P. contributed equally to this work.

Correspondence should be addressed to Dr. Elena Cattaneo, Department of Pharmacological Sciences and Centre for Stem Cell Research, Università degli Studi di Milano, Via Balzaretti 9, 20133 Milan, Italy. E-mail: elena.cattaneo@ unimi.it.

DOI:10.1523/JNEUROSCI.0917-10.2010

Copyright $\odot 2010$ the authors $\quad 0270-6474 / 10 / 3010844-07 \$ 15.00 / 0$
}

lying disease processes. Nevertheless, a valuable panel of transgenic and knock-in HD rodent models has been produced, allowing study of early- and late-stage changes (Menalled and Chesselet, 2002). Appearance of a specific dysfunction across multiple models may reinforce the potential relevance of such dysfunction in humans.

Recent studies in HD cells, mice, and patients indicated that reduced brain cholesterol biosynthesis may be a relevant phenotype in the human pathology (Sipione et al., 2002; Valenza et al., 2005, 2007a,b; Leoni et al., 2008). The underlying molecular mechanism involved diminished activity of sterol regulatory element binding proteins (SREBPs), master transcriptional regulators of several cholesterogenic genes (Valenza et al., 2005).

Brain cholesterol, almost all locally synthesized, is necessary for neuronal functions. Mice unable to properly synthesize cholesterol develop ataxia and tremor, and defective cholesterol homeostasis is often associated with neurological diseases (Valenza and Cattaneo, 2006). Here we demonstrate that cholesterol biosynthesis is reduced in brain samples from several rodent animals expressing mutant huntingtin and worsened in YAC mice carrying progressively increased CAG repeats. The sterol content is first reduced in HD synaptosomes and only at later stages in myelin. We also show that mRNA levels of cholesterol biosynthesis and efflux genes are reduced in HD astrocytes, leading to lowered cellular production and secretion of ApoE. Consistently, ApoE in the CSF of HD mice is associated with smaller lipoprotein particles, suggesting reduced cholesterol 
transport on ApoE-containing lipoproteins in vivo in presence of mutant huntingtin.

\section{Materials and Methods}

Animals and tissue isolation. Animals were cared for according to our institutional guidelines. After overnight starvation, the animals were killed and perfused with saline to avoid blood contamination, after which whole brains and areas were isolated. Fifty to seventy percent of the animals analyzed for each control (wt) and HD group was male, except for 21-month-old transgenic rat group (all females) and R6/2 group (all males). No gender-dependent variation in any of our parameters has been observed within each model. The number, the gender, and the CAG number of the animals used in the study are described in detail in supplemental Table S1 (available at www.jneurosci.org as supplemental material).

Analysis of cholesterol precursors, cholesterol, and 24OHC. Brain homogenates from YAC mice, Kin mice, and transgenic rats and homogenates from primary astrocytes were prepared in PBS. Fifty microliters of homogenates were added to a screw-capped vial sealed with a Teflon-lined septum, together with $100 \mathrm{ng}$ of 5a-cholest-7-en-3b-ol-1,2,5a,6a-d4 (D4-lathosterol; CDN Isotopes), $200 \mathrm{ng}$ of D324S-hydroxycholesterol, and $5 \mu \mathrm{g}$ of (D6) cholesterol internal standards. To prevent self-oxidation, $25 \mu \mathrm{l}$ of butylated hydroxytoluene (BHT) $(5 \mathrm{~g} / \mathrm{L})$ and $25 \mu$ lof EDTA $(10 \mathrm{~g} / \mathrm{L})$ were added to each vial, and argon was flushed through to remove air. Alkaline hydrolysis was allowed to proceed at room temperature $\left(22^{\circ} \mathrm{C}\right)$ for $1 \mathrm{~h}$ in the presence of $1 \mathrm{~m}$ ethanolic potassium hydroxide solution under magnetic stirring. After hydrolysis, the neutral sterols (cholesterol, lathosterol, and lanosterol) and oxysterol $(24 \mathrm{OHC})$ were extracted three times with $5 \mathrm{ml}$ of cyclohexane. The organic solvents were evaporated under a gentle stream of argon, and converted into trimethylsilyl ethers [pyridine:hexamethyldisilazane:trimethylchlorosilane, 3:2:1 (v/v/v)]. Gas chromatography-mass spectrometry (GC-MS) to quantify single components was performed as described by Valenza et al. (2007).

Myelin and synaptosome fractionation and sterol content measurement. Synaptosomes and myelin were obtained as described by Dodd et al. (1981), with some modifications. Briefly R6/2 $(n=4)$ and control wt mice $(n=4)$ for each time point (at 6 weeks and 12 weeks of age) were killed by cervical fracture and the whole brain was rapidly isolated and frozen at $-80^{\circ} \mathrm{C}$. After weighting of the tissue the whole brains were placed in 10 vol ice-cold unbuffered $0.32 \mathrm{M}$ sucrose, $\mathrm{pH} 7.0$, enriched with protease inhibitor cocktail (1:100, Sigma) and homogenized with a Teflon-glass homogenizer by 12 up and downs. Subsequent centrifugation steps were performed at $4^{\circ} \mathrm{C}$ in a Beckman L8 ultracentrifuge. Homogenate was centrifuged at $5100 \mathrm{rpm}$ for $10 \mathrm{~min}$ in the 70.1 TI-rotor (Beckman) to yield the crude nuclear pellet and the low-speed supernatant (interphase, S1). Eight milliliters of the S1 fraction were layered directly onto $4 \mathrm{ml}$ of $1.2 \mathrm{M}$ sucrose with protease inhibitor cocktail (Sigma) 1:100 and centrifuged at 50,000 rpm for $15 \mathrm{~min}$ in the 70.1 TI-rotor. Synaptosomes, myelin, and some microsomes were retained at the gradients interface. This material was collected carefully in a volume of $\sim 2 \mathrm{ml}$ and diluted with $5 \mathrm{ml}$ of ice-cold $0.32 \mathrm{M}$ sucrose, containing protease inhibitor cocktail (Sigma) 1:100, to a final volume of $7 \mathrm{ml}$. The diluted suspension was layered onto $4 \mathrm{ml}$ of $0.8 \mathrm{M}$ sucrose and protease inhibitor cocktail (Sigma) 1:100 and centrifuged under the same conditions as before. This yielded a synaptosome pellet ready for suspension, myelin was retained at the interface. To check the purity and clearness of each sub fraction, $50 \mu \mathrm{g}$ of proteins from myelin and synaptosome fractions and from the low-speed supernatant (S1) were separated on a $10 \%$ acrylamide gel and transferred to a nitrocellulose membrane. SNAP25 protein was detected using the mouse anti-Snap monoclonal antibody ( $1: 1000,2 \mathrm{~h}$, room temperature; $\mathrm{BD}$, Biosciences) while MBP protein was detected using the rat anti-MBP monoclonal antibody $(1: 500,2 \mathrm{~h}$ at room temperature; Millipore Bioscience Research Reagents), both followed by $1 \mathrm{~h}$ of incubation with secondary antibodies against mouse or rat IgG respectively labeled with horseradish peroxidase (1:3000 Bio-Rad Laboratories/1:2000 Zymes Laboratories). The membranes were then developed by chemiluminescence using the Super Signal West Femto Chemiluminescent Substrate (Pierce) for SNAP25 and Super Signal West Pico Chemiluminescent Substrate (Pierce) for MBP. The specific bands were detected at $25 \mathrm{kDa}$ and $20 \mathrm{kDa}$, respectively. Mouse $\alpha$-tubulin monoclonal antibody (1:1000; Sigma Aldrich) was used as the internal control.

Sterol levels were measured enzymatically in myelin and synaptosomes by means of solvent extraction [chloroform:methanol 3:2 (v/v) in presence of BHT $25 \mu \mathrm{g} / \mathrm{ml}$ ], and assayed by adding cholesterol esterase and oxidase, and using the Cholesterol Kit (FAR) as instructed by the manufacturer. The amounts of sterol were normalized on the basis of total protein content.

Culture of primary astrocytes. Brains from postnatal day 1 individual animals were placed into ice-cold HBSS (Invitrogen) containing $6 \mathrm{mg} / \mathrm{ml}$ glucose and $10 \mathrm{~mm}$ HEPES. Meninges were removed, striata and cortices were dissected, and cells were dissociated by repeated passage through a series of wide- to fine-bored pipettes. Dissociated cells were plated in DMEM (Invitrogen) with 10\% fetal bovine serum, 1\% mM L-glutamine (Invitrogen), and $1 \%$ of penicillin-streptomycin (Invitrogen) at one T75 flask per mouse. Cells were cultured in the presence of $5 \% \mathrm{CO}_{2}$ for $10 \mathrm{~d}$ with three medium changes. After reaching confluence, floating microglia was eliminated by shaking for $12 \mathrm{~h}$. Astrocytes were separated from attached microglia by mild trypsinization, counted, and reseeded. We prepared a total of three $w t$ and three R6/2 astrocyte cultures (each from genetically tested pups) from R6/2 mouse colony and nine wt and YAC128 (or YAC18) cultures of astrocytes from genetically tested YAC mouse colonies for the subsequent analyses of gene expression.

RNA isolation, retrotranscription, and real-time PCR for gene expression. Total RNA from astrocytes and P1 brains was isolated with RNeasy Mini Kit (Qiagen) and TRIZOL Reagent (Invitrogen), respectively. Genomic DNA was digested with DNase (Ambion). Total RNA $(0.25-0.5 \mu \mathrm{g})$ was reverse transcribed to single-stranded cDNA using Superscript III RNase $\mathrm{H}$ reverse transcriptase (Invitrogen) and random primers in a volume of $20 \mu \mathrm{l}$. Two independent reverse transcription (RT) reactions were set up for each RNA stock (corresponding to one individual pup). For each RT, two real-time PCR analyses were done in triplicate for each of the analyzed genes to obtain replicates for statistical analyses.

iCycler Thermal Cycler with Multicolor Real-time PCR Detection System (Bio-Rad) was used to evaluate gene expression in RNA from R6/2 astrocytes. All reactions were performed in a total volume of $25 \mu \mathrm{l}$ containing $2.5 \mathrm{ng}$ of cDNA, $50 \mathrm{~mm} \mathrm{KCl,} 20 \mathrm{~mm}$ Tris- $\mathrm{HCl}, \mathrm{pH} 8.4,0.2 \mathrm{~mm}$ dNTPs, $25 \mathrm{U} / \mathrm{ml}$ iTaq DNA polymerase, $3 \mathrm{~mm} \mathrm{MgCl}_{2}$, SYBR Green I, 10 nм fluorescein, stabilizers (iQ SYBR Green Supermix; Bio-Rad), and 0.2 $\mu \mathrm{M}$ forward and reverse primers. Amplification cycles consisted of an initial denaturing cycle at $95^{\circ} \mathrm{C}$ for $3 \mathrm{~min}$, followed by 45 cycles of $30 \mathrm{~s}$ at $95^{\circ} \mathrm{C}, 30 \mathrm{~s}$ at $59^{\circ} \mathrm{C}$ for hmgcr and $\beta$-actin, at $55^{\circ} \mathrm{C}$ for cyp 51 and $7 \mathrm{dhcr}$, and $30 \mathrm{~s}$ at $72^{\circ} \mathrm{C}$.

iCycler ABI 7500 fast (Applied Biosystems) was used to evaluate gene expression in RNA from YAC astrocytes. All reactions were performed in a total volume of $10 \mu \mathrm{l}$ containing $2.5 \mathrm{ng}$ of cDNA, SYBR Green I Dye, AmpliTaqGold DNA Polymerase, dNTPs, passive reference and optimized buffer components (Power SYBR Green PCR Master Mix, Applied Biosystems), and $0.2 \mu \mathrm{M}$ forward and reverse primers. Amplification cycles consisted of an initial cycle at $95^{\circ} \mathrm{C}$ for $10 \mathrm{~min}$ to activate the AmpliTaqGold Polymerase, followed by 40 cycles of $15 \mathrm{~s}$ at $95^{\circ} \mathrm{C}$ (denaturation step), $30 \mathrm{~s}$ at $60^{\circ} \mathrm{C}$ (annealing/extension step) for all genes.

In both cases, fluorescence was quantified during the annealing step, primer specificity and product formation was confirmed by a melting curve analysis $\left(55-94^{\circ} \mathrm{C}\right)$, and the amounts of target gene mRNA were normalized on $\beta$-actin as reference gene and by using the calibration curve method. The primer sequences used with Bio-Rad or Applied Biosystems iCyclers, respectively, are described in supplemental Table S2 (available at www.jneurosci.org as supplemental material).

Measurement of ApoE secretion. Astrocytes were seeded in six-well plates $(1,600,000$ per well) and, after $24 \mathrm{~h}$, exposed to serum-free DMEM/F12 (Invitrogen) for $8 \mathrm{~h}$. Conditioned medium was collected, centrifuged at $2000 \mathrm{rpm}$ for $4 \mathrm{~min}$, and then concentrated 10-fold by using Amicon Ultra-4 filter devices (Millipore). Cells were lysed in a buffer containing $10 \mathrm{~mm}$ Tris-HCl, pH 8, 1\% Triton-100 (Sigma), PMSF (1:250), and protease inhibitors (1:100, Sigma) and centrifuged at $10,000 \times g$ for $10 \mathrm{~min}$. Equal volumes of cell lysate samples and concentrated media (from the same cells) were separated on a $10 \%$ SDS- 


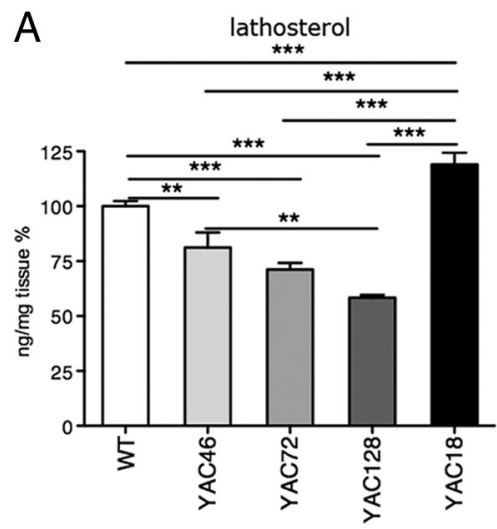

B

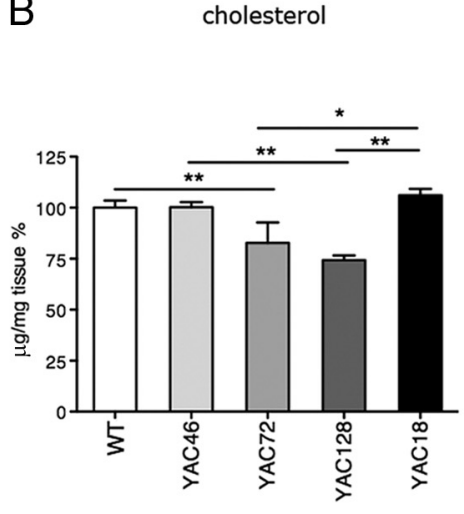

C

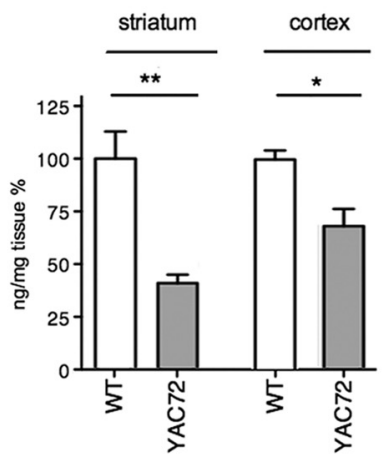

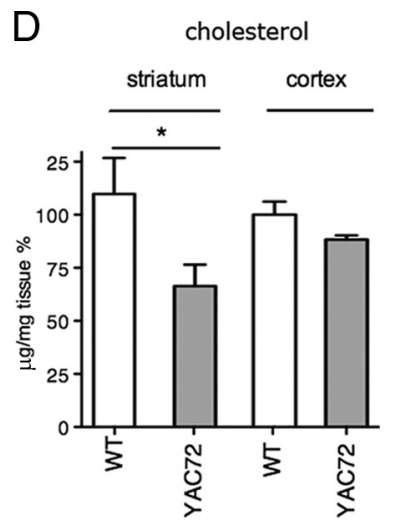

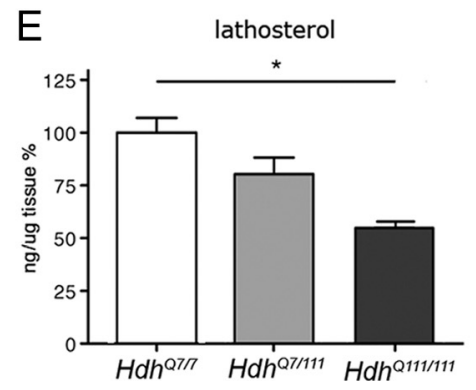

$\mathrm{F}$

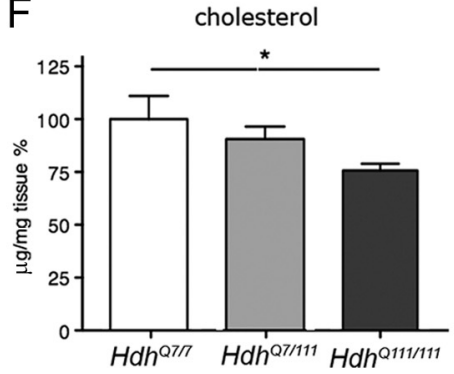

Figure 1. Reduced brain cholesterol biosynthesis in HD rodent models. Brain lathosterol $(\boldsymbol{A})$ and cholesterol $(\boldsymbol{B})$ levels were progressively reduced in 10-month-old YAC46 $(n=4)$, YAC72 $(n=4)$, and YAC128 $(n=4)$ and increased in YAC18 $(n=4)$ mice compared to wt mice $(n=4)$. Lathosterol $(C)$ and cholesterol (D) levels were reduced in striatum and cortex of YAC72 $(n=6)$ compared to wt mice $(n=6)$. Brain lathosterol $(\boldsymbol{E})$ and cholesterol $(\boldsymbol{F})$ levels were reduced in $H d h^{0111 / 111}(12$ months; $n=3)$ compared to $\operatorname{ddh}^{07 / 7}(n=5)$ and $\operatorname{Hdh}^{07 / 111}(n=5)$. All data were normalized above weight tissue. Graphs show mean as percentage above controls \pm SEM. ${ }^{*} p<0.05 ;{ }^{* *} p<0.01 ;{ }^{* * *} p<0.001$.

polyacrylamide gels and transferred to nitrocellulose membranes (Bio$\mathrm{Rad})$. The membranes were saturated with $5 \%$ nonfat milk and Trisbuffered saline with $0.1 \%$ Tween 20 and incubated at room temperature for $2 \mathrm{~h}$ with a goat anti-ApoE polyclonal antibody (AB947, 1:800; Millipore Bioscience Research Reagents), followed by $1 \mathrm{~h}$ at room temperature with a secondary antibody against goat IgG labeled with horseradish peroxidase (1:3000; Bio-Rad). Intracellular $\alpha$-tubulin levels, detected with a mouse $\alpha$-tubulin monoclonal antibody (1:5000; Sigma-Aldrich), were used to normalize measurements of cellular or secreted apoE. They were then developed by chemiluminescence using the Super Signal West Pico Chemiluminescent Substrate (Pierce). Densitometry analyses were made by using Quantity One (Bio-Rad). We also stained the filter membranes with Coomassie to reveal proteins level in the medium as loading control (one band at $\sim 75 \mathrm{kDa}$ was detected). For ApoE protein/medium analyses, samples from eight cultures generated from independent pups/genotype were used.

CSF collection. The meninges overlying the cisterna magna were exposed and cleaned in anesthetized animals. A 30 ga, 1/2 inch needle was used to puncture the arachnoid membrane covering the cisterna, and the CSF was collected with a polypropylene narrow-bore pipette over a period of $2 \mathrm{~min}$.

ApoE analysis in the CSF. Ten microliters of CSF per sample were mixed with $10 \times$ nondenaturing loading dye (bromophenol blue, $40 \%$ glycerol, and $1 \mathrm{~m}$ Tris, $\mathrm{pH} 6.8$ ), separated on $6 \%$ native-PAGE, transferred to PVDF membranes and exposed to primary antibody against ApoE (1:500, Santa Cruz Biotechnology). Blots were developed using Pico Super Signal Chemiluminescent Substrate (Pierce). Sizes of particles were determined by comparison with proteins of known molecular diameter in native high-molecular-weight marker (GE Healthcare).

Statistical analyses. The data were compared using Student's $t$ test or one-way ANOVA with Newman-Keuls multiple-comparison test. $p$ values, SEM, and means were calculated using GraphPad Prism version 4.0 .

\section{Results}

Brain cholesterol biosynthesis is reduced in HD models and is further diminished in mice carrying a progressively increased CAG repeat

Hydroxy-methyl-glutaryl-CoA-reductase (HMGCR) activity and cholesterol precursors levels were previously found reduced in the R6/2 and YAC128 mouse models (Valenza et al., 2007a and 2007b), starting from early disease stages. To assess whether the reduction of cholesterol biosynthesis could worsen with an increased CAG size, we analyzed cholesterol precursors by means of isotopic dilution mass spectrometry in whole brains from 10-months-old mice representing different YAC transgenic lines that overexpress full-length human huntingtin with 18 (YAC18), 46 (YAC46), 72 (YAC72), and 128 (YAC128) glutamine residues. Lathosterol levels were reduced in the brain from all mutant series (Fig. $1 A$ ), such that YAC128 < YAC72 < YAC46 < littermates (wt). YAC18 mice, instead, showed a 1.5-fold increase in lathosterol levels with respect to wt mice. Other cholesterol precursors (supplemental Fig. S1, available at www.jneurosci.org as supplemental material) and cholesterol itself (Fig. $1 B$ ) followed a similar progressive decrease in YAC mutant mice and an increase in YAC18 mice.

Previous results showed increased cholesterol content in striatum of YAC72 mice at 6-10-15 months of age with respect to wt mice, as measured by filipin staining and thin-layer chromatography (TLC) assay (Trushina et al., 2006). Conversely, we found that at 10 months of age cholesterol content was significantly reduced in striatum with a trend toward a decrease in the cortex (Fig. 1 D). Lathosterol levels were also reduced in YAC72 striatum and cortex (Fig. 1C) compared to wt samples.

Brain cholesterol precursors and cholesterol were similar in wt and presymptomatic HD rats (3 months of age) carrying a 


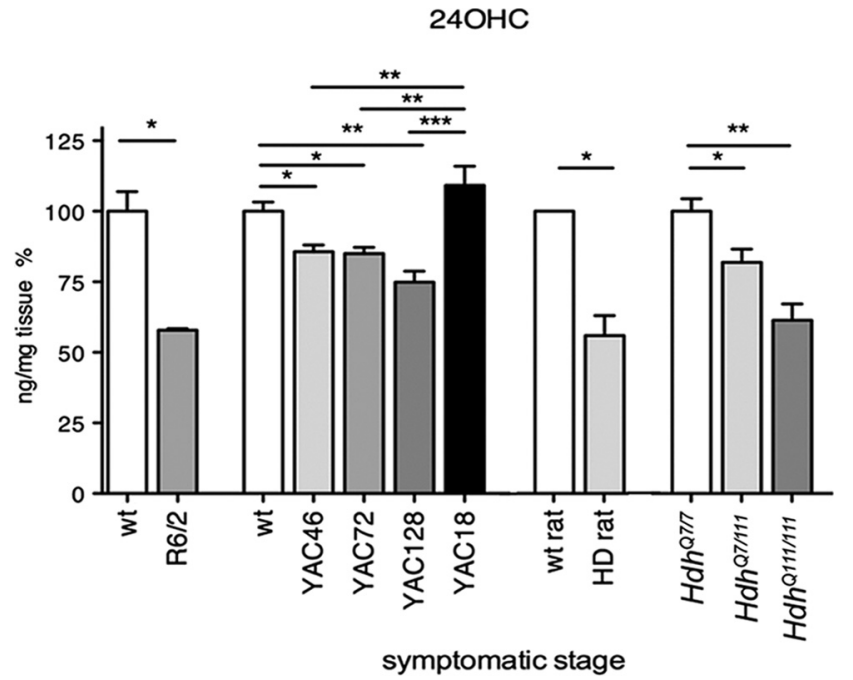

Figure 2. Reduced brain $240 \mathrm{HC}$ levels in HD rodent models. At the corresponding symptomatic stage, R6/2 (13 weeks; $n=3$ ), YAC (10 months; $n=4$ per group), $H d h^{07 / 111}$ (12 months; $n=5$ ), and $H d h^{0111 / 111}$ mice (12 months; $n=3$ ) and HD rats (21 months; $n=3$ ) showed reduced brain $240 \mathrm{HC}$ levels compared to their controls. All data were normalized above weight tissue. Graph shows mean as percentage above controls \pm SEM. ${ }^{*} p<0.05$; ${ }^{* *} p<0.01$; ${ }^{* * *} p<0.001$.

truncated huntingtin cDNA fragment with 51 CAG repeats (von Hörsten et al., 2003). However, symptomatic HD rats (21 months of age) showed 1.7- and 1.5-fold decrease in lathosterol (and in other precursors) and cholesterol compared with control rats (supplemental Fig. S2, available at www.jneurosci.org as supplemental material).

We next analyzed cholesterol precursors and cholesterol levels in heterozygous $\left(H d h^{Q 7 / 111}\right)$ and homozygous $\left(H d h^{Q 111 / 111}\right)$ knock-in mice carrying the CAG expansion within the mouse huntingtin gene (Wheeler et al., 1999). We found that brain lathosterol was progressively reduced in mutant mice at presymptomatic (3 months of age) (supplemental Fig. S3A, available at www.jneurosci.org as supplemental material) and symptomatic stage (12 months of age) (Fig. 1E) compared to littermates $(H d h Q 7 / 7)$, such that $H d h^{Q 7 / 7}>H d h^{Q 7 / 111}>H d h^{Q 111 / 111}$. Similarly, other cholesterol precursors (supplemental Fig. S3C-F, available at www.jneurosci.org as supplemental material) and cholesterol (Fig. $1 \mathrm{~F}$ ), albeit to a lesser extent, were also decreased.

We conclude that CAG mutation in huntingtin, when expressed in different contexts, leads to a dominant reduction in the overall cholesterol biosynthesis in YAC and knock-in mice and HD rats. This confirms finding on cholesterol precursors previously obtained in R6/2 mice (Valenza et al., 2007a), which is further substantiated by new evidence of reduced brain cholesterol content in 13-week-old R6/2 mice ( $n=3 ; 9.21 \pm 0.16 \mu \mathrm{g}$ of $\mathrm{chol} / \mathrm{mg}$ tissue) compared to age-matched wt mice $(n=3$; $12.44 \pm 1.36 \mu \mathrm{g}$ of $\mathrm{chol} / \mathrm{mg}$ tissue).

\section{Brain cholesterol turnover is reduced in HD models}

The main mechanism by which brain removes the daily excess of cholesterol is through its conversion into 24( S)-hydroxycholesterol (24OHC), a polar sterol capable of crossing the blood/brain barrier (BBB) (Björkhem and Meaney, 2004). Its measurement in plasma is believed to be an indicator of cholesterol turnover in the brain. Although with a different degree-possibly related to the severity of the initiating $\mathrm{HD}$ gene insult-24OHC levels were reduced in the brains of all HD animals compared to the respective controls (Fig. 2), with the exception of the YAC18 mice that exhibited mildly elevated $24 \mathrm{OHC}$ levels. We conclude that $24 \mathrm{OHC}$ levels are linked to, and mirror, reduced cholesterol biosynthesis in HD brains.

\section{Sterol content is primarily reduced in synaptosomes of HD mice}

To investigate whether the cholesterol biosynthesis reduction alters sterol content in myelin and/or in synaptosomes, we purified these fractions from whole brain of one of the HD models tested so far (R6/2 model) and analyzed sterol content. Western blot analyses against myelin binding protein (MBP) and SNAP25, markers of myelin and synaptosomes, respectively, assessed purity in each fraction (Fig. $3 A, B$ ). We found that at a stage before overt phenotypes (6 weeks of age) sterol content was already reduced in synaptosomes but not in myelin (Fig. 3C), while at the late-symptom stage (12 weeks of age), sterol content was reduced in both fractions (Fig. 3D).

These findings suggest that, in this model, early in the disease process, a compartment dedicated to the impulse transmission and neurotransmitter release carries suboptimal levels of sterols.

\section{Cholesterol biosynthesis is reduced in primary astrocytes from HD mice}

In adult brain, astrocytes are believed to supply mature neurons with additional required cholesterol for their neuronal functions (Björkhem and Meaney, 2004). We therefore analyzed the expression of key cholesterogenic genes, such as hmgcr, coding for the rate-limiting enzyme of cholesterol biosynthesis, Cyp51, an intermediate step gene, and 7-dehydroxy-cholesterol-reductase ( $7 \mathrm{dhcr}$ ), the last gene in the pathway, in primary astrocytes from R6/2, YAC128, and YAC18 pups and from their wt littermates. mRNA levels of the above genes were significantly reduced in primary R6/2 astrocytes with respect to wt astrocytes (Fig. 4A). Notably, mRNA levels of hmgcr were reduced of $\sim 20 \%$ in the brain of R6/2 pups at postnatal day 1 with respect to wt pups (supplemental Fig. S4, available at www.jneurosci.org as supplemental material). The defect in cholesterol biosynthesis is therefore traceable at early postnatal stages, suggesting that it may already be present in prenatal life. Similarly, primary YAC128 astrocytes showed reduced mRNA levels of hmgcr and $7 \mathrm{dhcr}$ (Fig. $4 B, C$ ) compared to wt astrocytes. Conversely, primary astrocytes from YAC18 mice showed a 1.3- and 2.1-fold increase of hmgcr and 7dhcr mRNA levels compared to wt and YAC128 astrocytes (Fig. $4 B$ ).

These findings indicate that cholesterol biosynthesis is reduced in astrocytes bearing either transgene-expressed fulllength or a short N-terminal piece of human mutant huntingtin.

\section{Genes involved in cholesterol efflux are reduced in HD astrocytes}

The synthesis and efflux of cholesterol in the brain are in equilibrium (Björkhem and Meaney, 2004). The ATP-binding cassette transporter 1 (ABCA1) plays a key role in facilitating cholesterol efflux from astrocytes through ApoE, providing mature neurons with the cholesterol required for their functions (HirschReinshagen et al., 2004; Karasinska et al., 2009). We found that mRNA levels of abcal and apoE were reduced in YAC128 astrocytes compared to wt astrocytes (Fig. $4 D, E$ ). The mRNA levels of Abcg4, encoding another ABC transporter highly expressed in the brain, were also reduced in YAC128 astrocytes (data not shown). Instead, mRNA levels for these genes were increased in YAC18 astrocytes (Fig. 4D,E). The content of cholesterol was similar in YAC128 and wt astrocytes $(10.3 \pm 1.1$ and $9.7 \pm 0.9 \mu \mathrm{g}$ 
of chol/ $\mu \mathrm{g}$ of prot, respectively), suggesting that mRNA levels of cholesterol efflux genes were reduced as a consequence of reduced cholesterol biosynthesis.

ApoE secretion is reduced in vitro and ApoE-containing lipoproteins are reduced in size in YAC 128 mice

To assess whether the decrease of abcal and $a p o E$ mRNA levels in mutant astrocytes led to reduced ApoE secretion, primary astrocytes were exposed to serumfree medium for $8 \mathrm{~h}$ followed by analyses of the levels of cellular and secreted ApoE. Cellular levels of ApoE were reduced in YAC128 astrocytes compared to wt astrocytes (Fig. 4F). Similarly, the medium conditioned by YAC128 astrocytes contained lower levels of ApoE with respect to the medium conditioned by wt astrocytes (Fig. 4G). Even if ApoE is reduced in HD astrocytes, ApoE-containing lipoproteins could still be efficiently lipidated in vivo resulting in normal size particles. We therefore analyzed the size of ApoE-containing lipoproteins in the CSF from YAC128 and wt mice by means of non-denaturing gel electrophoresis. In the YAC128 CSF ApoE associated with lipoproteins smaller than those of between 10 and $17 \mathrm{~nm}$ in diameter in wt CSF (Fig. 4H), suggesting reduced cholesterol transport on ApoEcontaining lipoproteins circulating in the brain of mutant mice, likely contributing to HD pathogenesis.

\section{Discussion}

Here we report that brain cholesterol precursors and cholesterol levels are remarkably reduced in multiple HD rodent models and that mRNA levels of hmgcr are already diminished in the R6/2 mouse model at postnatal day 1 . The data of the different YAC mice also indicate that cholesterol deficit worsen with the increase of CAG repeats. This is particularly true for YAC46 and YAC72 mice since they express human huntingtin at equivalent levels, approximately one-half of endogenous huntingtin expression (Hodgson et al., 1999). The expression levels of human huntingtin in YAC128 and YAC18 mice used in the current study are instead 1.5- to 1.7-fold higher than those of endogenous huntingtin (Valenza et al., 2007) and show a robust decrease (in YAC128) or increase (in YAC18). Our data in the $H d h^{Q 7 / 111}$ versus $H d h^{Q 111 / 111}$ mice and in the YAC18 transgenic mice also suggest that, in the adult brain, the dose of the non-HD-expanded huntingtin protein may increase cholesterol biosynthesis.

The apparent contradiction between our results in YAC72 mice and Trushina et al. (2006) data in the same animals, showing increased free cholesterol accumulation in striata of agematched YAC72 mice, may be due to the different method that we used, i.e., isotopic dilution mass spectrometry, a more sensitive analytical method than filipin staining and thin-layer chromatography, used by Trushina et al. (2006). Another difference may be in the way animals were treated before analyses. In our case, animals were perfused with saline to avoid blood contamination, which might interfere with the sterol quantification.

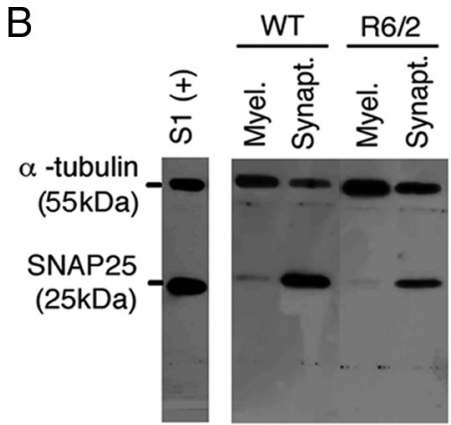

D

12 weeks of age sterol content

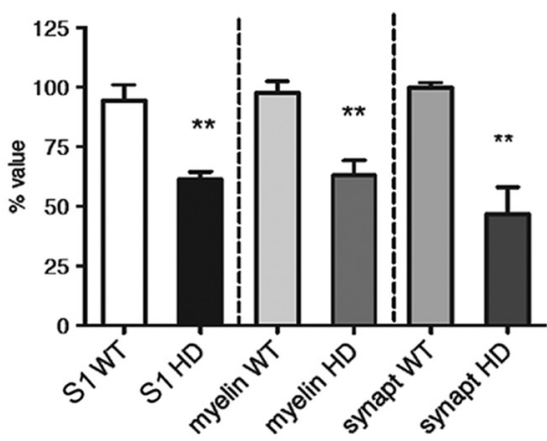

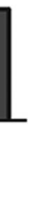

weeks of age sterol content

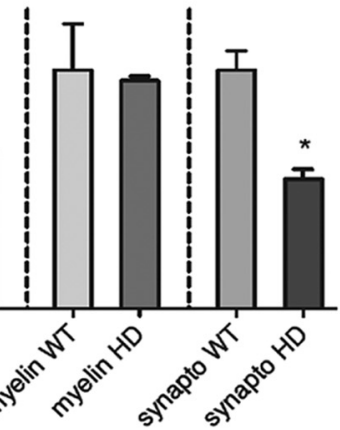

Figure 3. Decreased sterol content in R6/2 synaptosomes and myelin. $A, B$, Representative Western blot in myelin and synaptosome at 12 weeks of age $(\boldsymbol{D})$ in $R 6 / 2$ compared to wt samples. Data were normalized on protein content. Graphs show the mean of three experiments (per age) as percentage above respective controls \pm SEM. ${ }^{*} p<0.05 ;{ }^{* *} p<0.01$.

The data reported so far could be also relevant for biomarkers research. In the brain, excretion of exceeding cholesterol occurs primarily after conversion to 24OHC (Björkhem and Meaney, 2004). Therefore, plasma $24 \mathrm{OHC}$ may be an indicator of cholesterol homeostasis within the brain. Our previous data showed that brain-derived $24 \mathrm{OHC}$ was reduced in plasma from HD patients at different disease stages (Leoni et al., 2008) and in brain and blood from YAC128 mice (Valenza et al., 2007). We now report that $24 \mathrm{OHC}$ levels are reduced in the brain of all $\mathrm{HD}$ rodent models herein tested, supporting further studies aimed at evaluating $24 \mathrm{OHC}$ as a candidate biomarker in HD.

Brain cholesterol is involved in synaptogenesis and neurotransmitter release (Thiele et al., 2000, Mauch et al., 2001). R6/2 transgenic mice exhibited a lower content of sterols in isolated synaptosomes, which may contribute to the synaptic dysfunction described in HD models (Smith et al., 2005). Cholesterol biosynthesis also plays a key role in myelin formation. Imaging and biochemical studies have confirmed progressive loss of white matter in HD patients already 10 years before the overt symptoms onset (Paulsen et al., 2006). However, isolated myelin from R6/2 mouse brains showed lowered sterol content only at overt symptomatic stages. Increased transport of maternal cholesterol to the embryo and fetus (Yoshida and Wada, 2005) or increased uptake from periphery might protect myelin from a reduced sterol content in R6/2 newborns.

Adult neurons are thought to reduce or even abandon cholesterol biosynthesis (Pfrieger, 2003) and rely on the delivery of cholesterol from astrocytes through ApoE, the most important lipoprotein in the brain (Mauch et al., 2001). In this supposed 


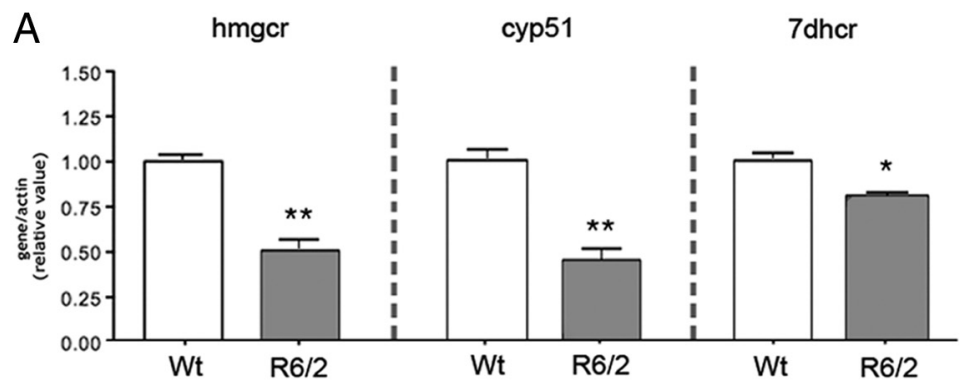

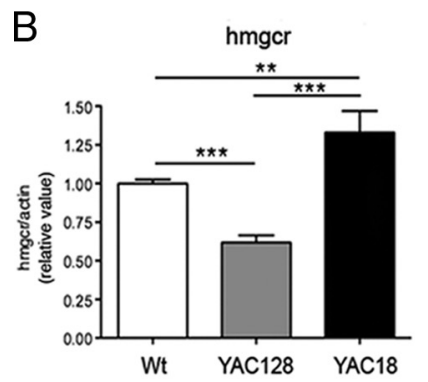

$\mathrm{F}$ cellular ApoE
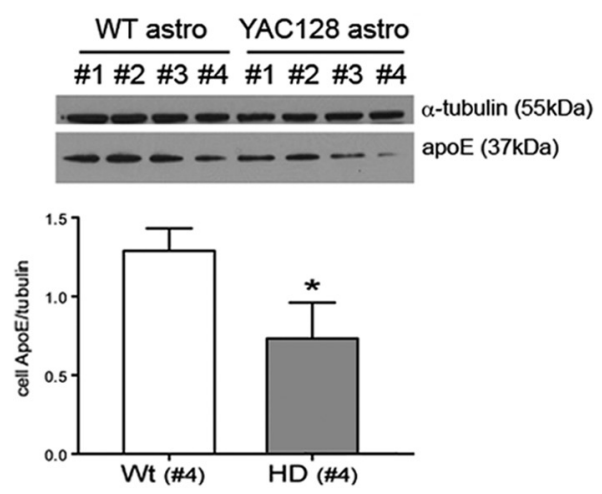
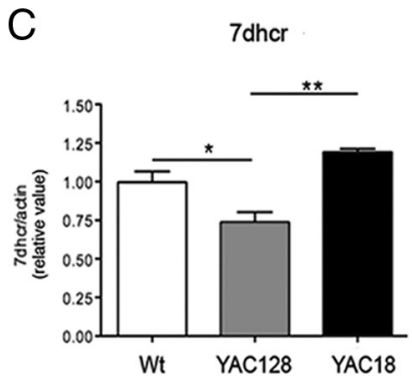

$\mathrm{G}$
D

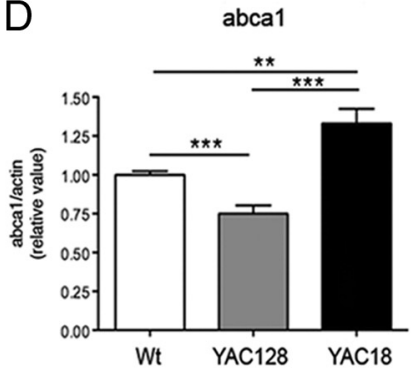

medium ApoE
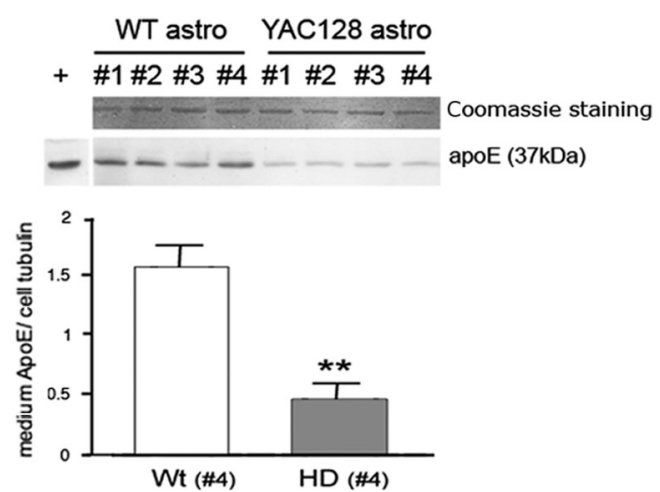

E

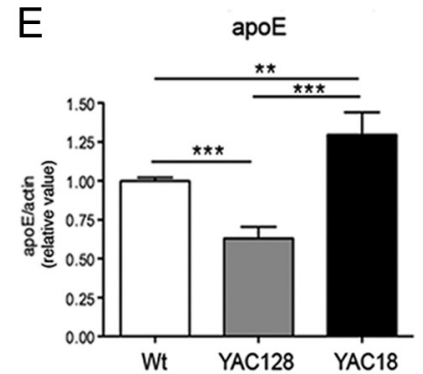

$\mathrm{H}$ CSF ApoE-particles

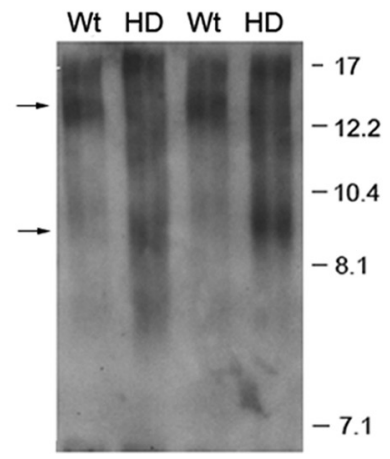

Figure 4. Reduced ApoE secretion in HD astrocytes and smaller ApoE-containing lipoproteins in HD CSF. A, Hmgcr, cyp51, and 7dhcr mRNA levels were reduced in primary R6/2 astrocytes with respect to wt astrocytes. Each column represents the mean from six Real-time PCR for each of the three independent cultures of R6/2 and wt astrocytes. B-E, Hmgcr, 7 dhcr, abca 1, and apoE mRNA levels were reduced in primary YAC128 astrocytes and increased in primary YAC18 compared to wt astrocytes. Each column corresponds to the mean of six real-time PCR performed on each of the nine independent cultures of YAC128/YAC18/wt astrocytes. All data $(\boldsymbol{A}-\boldsymbol{E})$ were normalized on $\beta$-actin. Graphs show mean of fold change above controls \pm SEM. $\boldsymbol{F}, \boldsymbol{G}$, Representative Western blots and densitometry for ApoE in whole-cell lysate $(\boldsymbol{F})$ and in conditioned medium $(\boldsymbol{G})$ from primary wt and YAC128 astrocytes showed reduced ApoE levels in YAC128 samples $(n=4)$ compared to wt samples $(n=4)$. Cellular $\alpha$-tubulin was used to normalize variations in total cell number in measurements of secreted ApoE. Coomassie staining of the membranes corresponding to proteins in the medium (4G) was also used as loading control. Graphs show mean \pm SEM. $\boldsymbol{H}$, A representative Western blot for ApoE in non-denaturing conditions shows smaller ApoE-containing-lipoproteins in CSF samples from YAC128 mice (HD; $n=2)$ compared to wt samples $(n=2)$, as indicated by arrows. Four of six CSF samples from individual YAC128 mice analyzed showed smaller ApoE-containinglipoproteins compared to the same number of wt mice. ${ }^{*} p<0.05$; ${ }^{* *} p<0.01$; ${ }^{* * *} p<0.001$.

loop, we found that cholesterol metabolism in astrocytes was compromised at several levels. First, mRNA levels of cholesterol biosynthesis and efflux genes were reduced in primary astrocytes bearing mutant huntingtin. One of the mechanisms underlying reduced cholesterol biosynthesis in mutant astrocytes may involve SREBPs, the transcription factors regulating almost all cholesterogenic genes, whose activity is reduced in HD cells and tissues through a mechanism occurring outside the nucleus (Valenza et al., 2005). Secondarily, mutant astrocytes produced and secreted less ApoE. Thirdly, ApoE-containing lipoproteins resulted smaller in size in the CSF from YAC128 mice with respect to CSF from wt mice, suggesting reduced cholesterol transport on ApoE-containing lipoproteins in vivo. All these findings indicate that non-cell-autonomous mechanisms involving astrocytes may determine the cholesterol phenotype observed in HD brains. These data do not exclude that other cell types may contribute to the generalized cholesterol defect observed in vivo.
Reduced brain cholesterol precursors and 24OHC in HD animal models might also lead to reduced activity of liver X receptors (LXRs), with consequences on LXR-mediated transcription of $a b c a 1$ and $a p o E$, and ultimately contributing, along with reduced SREBP activation, to cholesterol phenotype in HD. Notably, treatment with a LXR agonist partially rescues huntingtin knockdown phenotypes in zebrafish (Futter et al., 2009), suggesting that modulation of cholesterol efflux might be an alternative strategy for targeting cholesterol-dependent neuronal damage in HD.

Although further basic research is needed to understand how cholesterol deregulation impairs neuronal cell functions, these data consolidate the hypothesis of a cholesterol reduction in the brains of HD models. On the whole, four different HD rodent models - for a total of $\sim 90$ transgenic and knock-in animalsshow this phenotype compared to $>100$ controls tested so far (Valenza et al., 2005, 2007ab; this paper). It will be interesting to 
investigate routes to increase the activity of cholesterol biosynthesis or efflux pathways in HD brain, although it is unclear whether augmenting brain cholesterol will be sufficient to protect debilitated neurons. Novel strategies to selectively and finely modulate brain cholesterol metabolism will be necessary to define the role of altered cholesterol metabolism in HD pathogenesis.

\section{References}

Björkhem I, Meaney S (2004) Brain cholesterol: long secret life behind a barrier. Arterioscler Thromb Vasc Biol 24:806-815.

Dodd PR, Hardy JA, Oakley AE, Edwardson JA, Perry EK, Delaunoy JP (1981) A rapid method for preparing synaptosomes: comparison with alternative procedures. Brain Res 226:107-118.

Futter M, Diekmann H, Schoenmakers E, Sadiq O, Chatterjee K, Rubinsztein DC (2009) Wild-type but not mutant huntingtin modulates the transcriptional activity of liver X receptors. J Med Genet 46:438-446.

Hirsch-Reinshagen V, Zhou S, Burgess BL, Bernier L, McIsaac SA, Chan JY, Tansley GH, Cohn JS, Hayden MR, Wellington CL (2004) Deficiency of ABCA1 impairs apolipoprotein E metabolism in brain. J Biol Chem 279:41197-41207.

Hodgson JG, Agopyan N, Gutekunst CA, Leavitt BR, LePiane F, Singaraja R, Smith DJ, Bissada N, McCutcheon K, Nasir J, Jamot L, Li XJ, Stevens ME, Rosemond E, Roder JC, Phillips AG, Rubin EM, Hersch SM, Hayden MR (1999) A YAC mouse model for Huntington's disease with full-length mutant huntingtin, cytoplasmic toxicity, and selective striatal neurodegeneration. Neuron 23:181-192.

Karasinska JM, Rinninger F, Lütjohann D, Ruddle P, Franciosi S, Kruit JK, Singaraja RR, Hirsch-Reinshagen V, Fan J, Brunham LR, Bissada N, Ramakrishnan R, Wellington CL, Parks JS, Hayden MR (2009) Specific loss of brain ABCA1 increases brain cholesterol uptake and influences neuronal structure and function. J Neurosci 29:3579-3589.

Leoni V, Mariotti C, Tabrizi SJ, Valenza M, Wild EJ, Henley SM, Hobbs NZ, Mandelli ML, Grisoli M, Björkhem I, Cattaneo E, Di Donato S (2008) Plasma 24S-hydroxycholesterol and caudate MRI in pre-manifest and early Huntington's disease. Brain 131:2851-2859.

Mauch DH, Nägler K, Schumacher S, Göritz C, Müller EC, Otto A, Pfrieger FW (2001) CNS synaptogenesis promoted by glia-derived cholesterol. Science 294:1354-1357.

Menalled LB, Chesselet MF (2002) Mouse models of Huntington's disease. Trends Pharmacol Sci 23:32-39.

Paulsen JS, Magnotta VA, Mikos AE, Paulson HL, Penziner E, Andreasen NC, Nopoulos PC (2006) Brain structure in preclinical Huntington's disease. Biol Psychiatry 59:57-63.

Pfrieger FW (2003) Outsourcing in the brain: do neurons depend on cholesterol delivery by astrocytes? Bioessays 25:72-78.

Sipione S, Rigamonti D, Valenza M, Zuccato C, Conti L, Pritchard J, Kooper- berg C, Olson JM, Cattaneo E (2002) Early transcriptional profiles in huntingtin-inducible striatal cells by microarray analyses. Hum Mol Genet 11:1953-1965.

Smith R, Brundin P, Li JY (2005) Synaptic dysfunction in Huntington's disease: a new perspective. Cell Mol Life Sci 62:1901-1912.

The Huntington's Disease Collaborative Research Group (1993) A novel gene containing a trinucleotide repeat that is expanded and unstable on Huntington's disease chromosomes. Cell 72:971-983.

Thiele C, Hannah MJ, Fahrenholz F, Huttner WB (2000) Cholesterol binds to synaptophysin and is required for biogenesis of synaptic vesicles. Nat Cell Biol 2:42-49.

Trushina E, Singh RD, Dyer RB, Cao S, Shah VH, Parton RG, Pagano RE, McMurray CT (2006) Mutant huntingtin inhibits clathrin-independent endocytosis and causes accumulation of cholesterol in vitro and in vivo. Hum Mol Genet 15:3578-3591.

Valenza M, Cattaneo E (2006) Cholesterol dysfunction in neurodegenerative diseases: is Huntington's disease in the list? Prog Neurobiol 80: 165-176.

Valenza M, Rigamonti D, Goffredo D, Zuccato C, Fenu S, Jamot L, Strand A, Tarditi A, Woodman B, Racchi M, Mariotti C, Di Donato S, Corsini A, Bates G, Pruss R, Olson JM, Sipione S, Tartari M, Cattaneo E (2005) Dysfunction of the cholesterol biosynthetic pathway in Huntington's disease. J Neurosci 25:9932-9939.

Valenza M, Leoni V, Tarditi A, Mariotti C, Björkhem I, Di Donato S, Cattaneo E (2007a) Progressive dysfunction of the cholesterol biosynthesis pathway in the R6/2 mouse model of Huntington's disease. Neurobiol Dis 28:133-142.

Valenza M, Carroll JB, Leoni V, Bertram LN, Björkhem I, Singaraja RR, Di Donato S, Lutjohann D, Hayden MR, Cattaneo E (2007b) Cholesterol biosynthesis pathway is disturbed in YAC128 mice and is modulated by huntingtin mutation. Hum Mol Genet 16:2187-2198.

von Hörsten S, Schmitt I, Nguyen HP, Holzmann C, Schmidt T, Walther T, Bader M, Pabst R, Kobbe P, Krotova J, Stiller D, Kask A, Vaarmann A, Rathke-Hartlieb S, Schulz JB, Grasshoff U, Bauer I, Vieira-Saecker AM, Paul M, Jones L, et al. (2003) Transgenic rat model of Huntington's disease. Hum Mol Genet 12:617-624.

Vonsattel JP (2008) Huntington disease models and human neuropathology: similarities and differences. Acta Neuropathol 115:55-69.

Wheeler VC, Auerbach W, White JK, Srinidhi J, Auerbach A, Ryan A, Duyao MP, Vrbanac V, Weaver M, Gusella JF, Joyner AL, MacDonald ME (1999) Length-dependent gametic CAG repeat instability in the Huntington's disease knock-in mouse. Hum Mol Genet 8:115-122.

Yoshida S, Wada Y (2005) Transfer of maternal cholesterol to embryo and fetus in pregnant mice. J Lipid Res 46:2168-2174.

Zuccato C, Valenza M, Cattaneo E (2010) Molecular mechanisms and potential therapeutical targets in Huntington's disease. Physiol Rev 90:905981. 